\title{
An Engineer's View of Hair Cell Function: A Theory of Capacitive Transduction
}

\author{
Michelangelo Rossetto \\ 125 w 86 st, New York, NY, USA \\ Email: marossetto@libero.it
}

Received 2 March 2015; accepted 20 March 2015; published 24 March 2015

Copyright (C) 2015 by author and Scientific Research Publishing Inc.

This work is licensed under the Creative Commons Attribution International License (CC BY). http://creativecommons.org/licenses/by/4.0/

c) $\underset{\mathrm{EY}}{\mathrm{P}}$ Open Access

\begin{abstract}
Using an engineering perspective which is looking at the hair cells cilia as a total entity interacting with its neighboring cilia along their entire length, a theory of capacitive transduction is developed. This larger scaled view of cilia interaction suggests that transduction may be achieved through a mechanically controlled variable capacitor in the cilia bundle. A brief review of some seldom considered facts about electrical capacitors supports the hypothesis presented. Experimental measurements of hair cell reversal potential and ionic conditions surrounding the cilia during transduction, long reported in the biophysical literature, also support a capacitive hypothesis of transduction.
\end{abstract}

\section{Keywords}

Hair Cell, Cilia, Transduction, Nernst

\section{Introduction}

The hair cell, shown schematically in Figure 1, has been subjected to intensive investigation and while a wealth of data has accumulated on the biophysics of the sensory cell [1], there are some questions about the interpretation of this data. The transduction mechanism, long assumed to be implemented by a mechanically controlled ion channel, is falsified by measurements in the hair cell literature. The measured behavior of the hair cell is not consistent with the demands of the Nernst equation which defines the behavior of an ion channel in a membrane separating ionic solutions of different concentrations [2]. Although it is customary to think of cellular function in terms of membrane processes such as ion channels and ion pumps, engineers, being trained to focus on mechanisms from a structural perspective, tend to look for larger scaled mechanical and electrical mechanisms. In this case, it leads to the hypothesis that hair cell transduction is achieved with a capacitive transducer. This paper presents a new interpretation of the mechanism of mechanical to electrical transduction in the hair cell based 


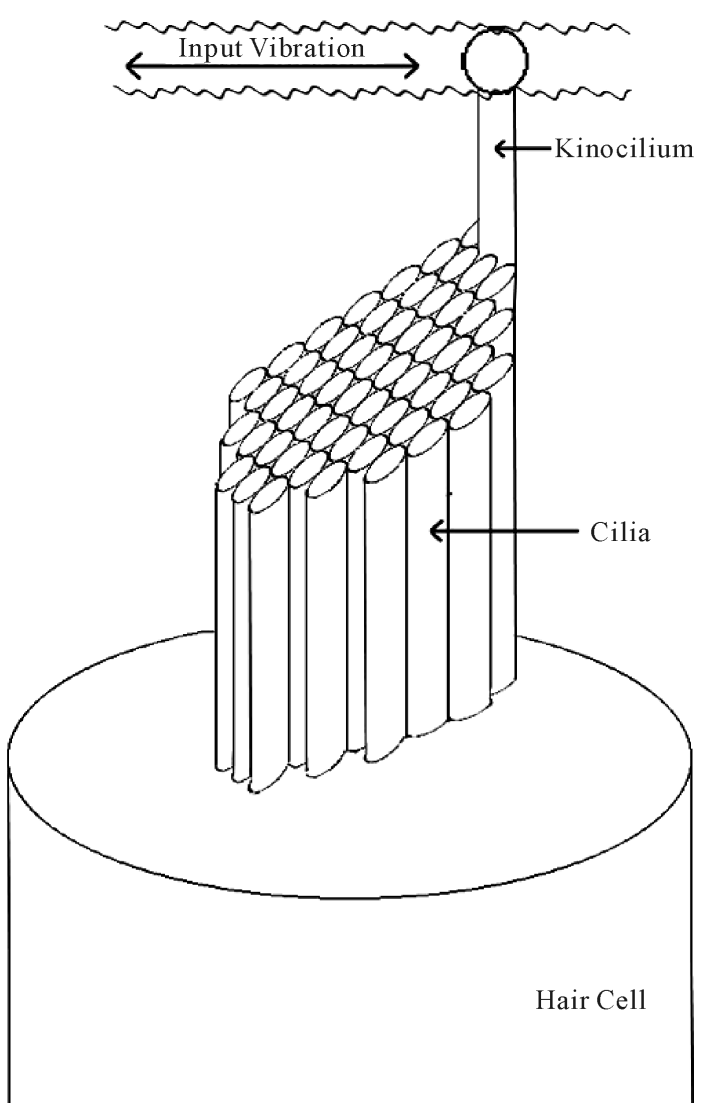

Figure 1. The active, ciliated, end of the hair cell drawn schematically showing how the bundle of cilia relate to the kinocilium which transfers the input vibration to the cilia.

on the mechanical modulation of the spacing between adjacent cilia, and consequently, the membrane capacity of the hair cell cilia by the motion of the cilia during acoustic stimulation.

\section{Capacitive View of Hair Cell Transduction}

The hair cell can be viewed as a capacitive transducer. That is to say that the mechanical to electrical transduction is the result of a mechanically driven variable capacitor. The variable capacitor results from the interaction of adjacent cilia along their total length resulting in the variation of the membrane capacity of the cilia. The mechanical input, by alternately pressing together and pulling apart the relatively stiff cilia over their whole length, varies the area of the cilia membrane exposed to the extra cellular space. Figure 2 shows the two conditions that can exist between adjacent cilia. Figure 2(a) shows a cross section of two cilia pulled away from each other. All of the cilia is exposed to the extra cellular fluid. Figure 2(b) shows two adjacent cilia pressed together. Here some of the cilia membrane is not exposed to the extra-cellular fluid. The membrane capacity of the cilia will be reduced in the same proportion as the extra-cellular exposure of the membrane is reduced. The reduction of exposure of the membrane does not require actual contact of the two cilia, but begins when the ionic double layer which surrounds the cilia membrane [3] begins to be squeezed out. This will occur as the Deby length for ringers, approximately 5 to 8 angstroms, is approached.

\section{Explanation of Capacitance}

In order to understand how a capacitive hair cell transducer works, it is necessary to briefly review the physical and electrical relations in an electrical capacitor. Figure 3 is a schematic representation of an electrical capacitor. Some pertinent factors are: 


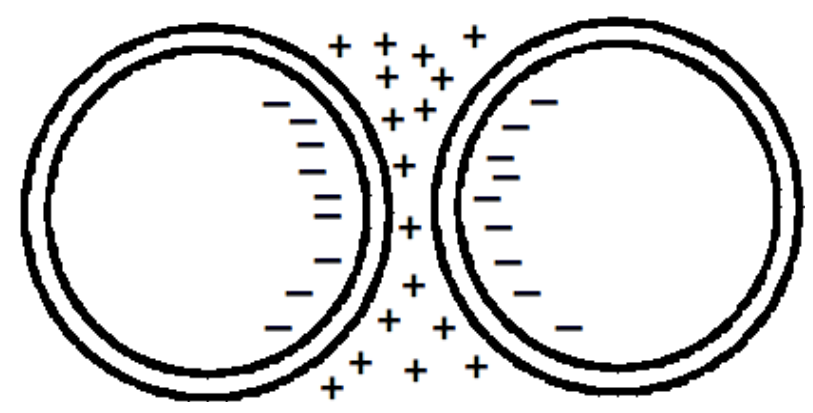

(a)

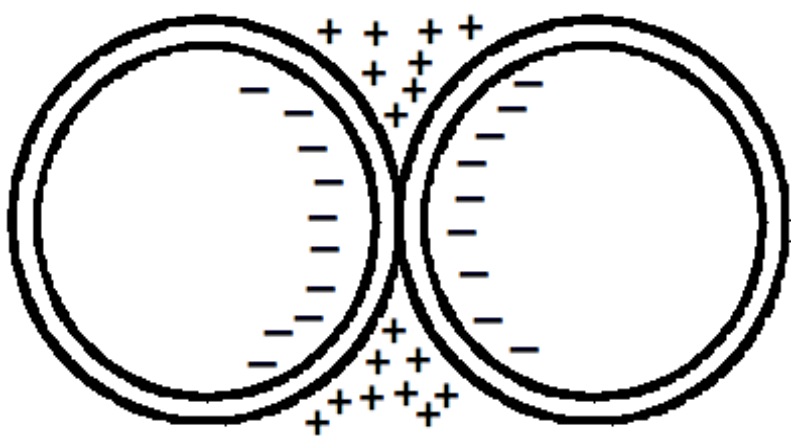

(b)

Figure 2. A cross section of the area of contact between two adjacent cilia is shown under two different conditions. (a) The two cilia are pulled apart. This allows the external positively polarized endolymph to contact all of the cilia surface. The separation distance is limited by side links; (b) The two cilia are pressed together showing an area of contact between the cilia which is consequently not in contact with the external ionic fluid. The membrane area exposed to the endolymph has been reduced and consequently the membrane capacity of the cell is reduced.

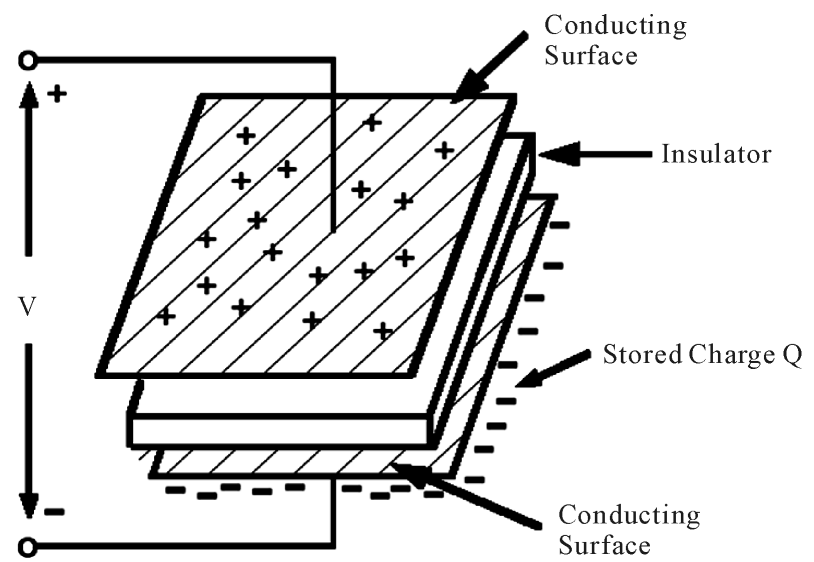

Figure 3. Schematic representation of an electrical capacitor.

1) An electrical capacitor is formed by two conducting surfaces separated by an insulator. In the cell the insulator is the lipid bilayer and the conducting surfaces are formed by the adjacent ionic fluids without regard to which ions are present in the ionic fluid.

2) The capacity $C$ in farads is the amount of charge $Q$ in coulombs the capacitor can store per volt across the capacitor. 


$$
C=\frac{Q}{V}
$$

3) The capacity is proportional to the conducting surface.

4) If the capacity is changed without change in the stored charge $Q$ the voltage must change to satisfy Equation (1).

5) The magnitude of small changes in $V$ and $C$ with $Q$ constant are expressed by:

$$
\Delta V=\frac{\Delta C}{C} V
$$

6) There is no delay to a change in voltage due to a change in capacity with a constant $Q$. Voltage change results from redistribution of charge. This redistribution of charge, an instantaneous process for a perfect conductor is, very fast in the ionic solutions found in biological systems.

\section{Endolymph}

The endolymph, which is the fluid bathing the hair cell cilia in the cochlea, carries a positive polarizing voltage. The utility of the positive endolymph voltage has long evaded explanation. A positive voltage is a rarity in biological systems. This suggests that there must be a serious function for this unusual potential to perform. The positive endolymph voltage begins to make sense in the context of capacitive transduction. The positive endolymph voltage directly enhances the sensitivity of the mechanical to electrical transduction. Figure 4, which is a schematic representation of the electrical circuit equivalent of distinct cellular areas, shows that the variable capacity of the cilia is polarized on one side by the $-60 \mathrm{mV}$ found inside the cell body. On the other side, the endolymph is polarized to $+70 \mathrm{mV}$. The cilia variable capacitor operates in a voltage field of $130 \mathrm{mV}$, the sum of these voltages. This is an unusually high potential in biology. We see, recalling Equation (2), that the voltage produced by the transduction is proportional to this composite polarizing voltage. The cell body meanwhile is isolated from the positive potential by a system of tight junctions between adjacent hair cells, and has a normal environment of $-60 \mathrm{mV}$ inside and $0 \mathrm{mV}$ outside. Only the cilia side of the cell is exposed to the endolymph with its positive polarizing voltage.

\section{Example}

The dimensions of a typical hair cell listed above are used in the following example. When cilia are moved from a position of no contact as shown in Figure 2(a) to a condition of approximately $10 \%$ contact as shown in Figure 2(b), there is a reduction in membrane exposed to the endolymph. In this example the reduction in area $\Delta A$ is about 80 square microns out of a total $(A)$ of about 3200. Applying Equation (2) where we may substitute $\frac{\Delta A}{A}$ for $\frac{\Delta C}{C}, \Delta V=\frac{\Delta A}{A} V=(80 / 3200) 130$. This gives $\Delta V=3.2 \mathrm{mV}$. This is a $5 \%$ change in the $60 \mathrm{mV}$ resting potential of the cell body. This demonstrates the utility of the positive polarization of the endolymph. Without the positive polarization the stimulus would have produced a signal of only $1.5 \mathrm{mV}$.

\section{Supporting Cells}

Hair cells require precise structural positioning in the sensory organs to preform their function. They are usually positioned by specialized supporting cells which envelope part, and in some cases all, of the cell body tightly. In places where the supporting cells contact the cell body the membrane capacity is cut in half by virtue of there being two lipid bilayers between the conducting ionic solutions which define the membrane capacity. This reduction in the capacitance of the cell body reduces the denominator on the right side of Equation (2) and hence enhances the signal in the hair cell by increasing the magnitude of the term $\frac{\Delta C}{C}$.

\section{Ionic Environment}

If transduction is accomplished, as hypothesized in this paper, via a modulation of cilia electrical capacitance, it should be independent of which ion is present in the extra-cellular conducting medium. This independence of 


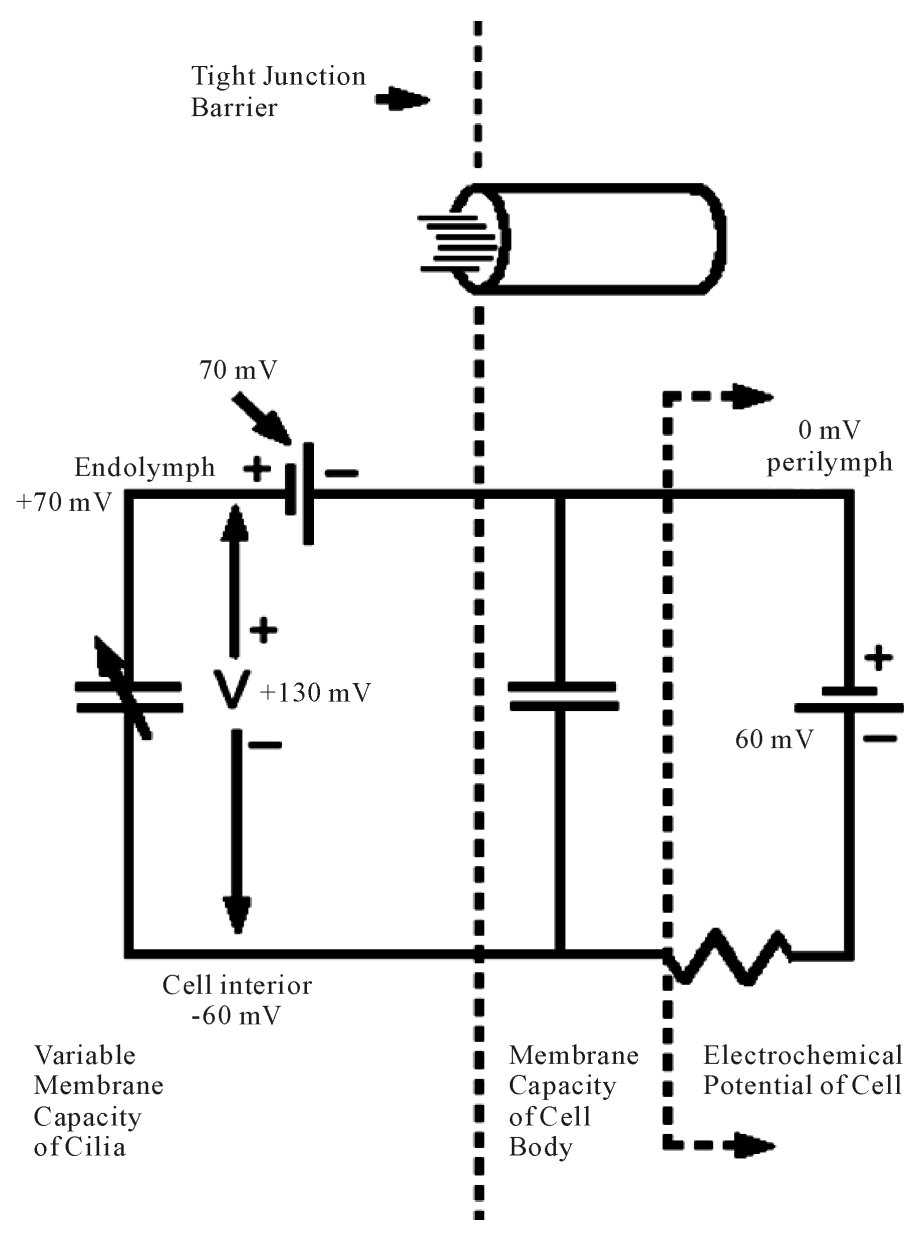

Figure 4. Schematic representation of the capacity of the hair cell. The membrane of the cell body and the cilia are one continuous surface but are shown separated here to illustrate how the two parts of the cell and their membrane capacity are polarized with different potentials. The cell body is polarized with the $60 \mathrm{mV}$ provided by customary cellular processes. The top of the cell including the cilia is polarized with the $-60 \mathrm{mV}$ inside while the outside is in contact with the endolymph which carries a potential of up to $+70 \mathrm{mV}$. The cilia membrane therefore sees a total potential, in this example of $130 \mathrm{mV}$, the sum of these two voltages. The cilia end of the cell, which is in contact with the endolymph at $+70 \mathrm{mV}$, is isolated from the cell body, which is in contact with the perilymph at $0 \mathrm{mV}$, by system of tight junctions. A typical cell body has 50 cilia of average length of $5 \mathrm{u}$ and a diameter of approximately $1 \mathrm{u}$. while a typical cell body has a diameter of $20 \mathrm{u}$ and a length of $30 \mathrm{u}$.

transduction to ionic environment has been reported in the hair cell biophysical literature. Transduction has been observed with a variety of cations bathing the hair cell cilia [4]. They observed transduction with $\mathrm{Li}^{+}, \mathrm{Na}^{+}, \mathrm{K}^{+}$, $\mathrm{Rb}^{+}, \mathrm{Cs}^{+}, \mathrm{NH}_{4}^{+}$, and even the organic ion tetramethylammonium.

\section{Reversal Potential}

The reversal potential, which can deduced from Equation (2), should always occur at zero volts since it is not dependent on the equilibrium potential of an ion, but is dependent on the voltage term " $V$ " on the right side of the equation. Transduction reversal potential, shown schematically in Figure 5, has been repeatedly found to occur with a reversal value of zero [4] [5]. 


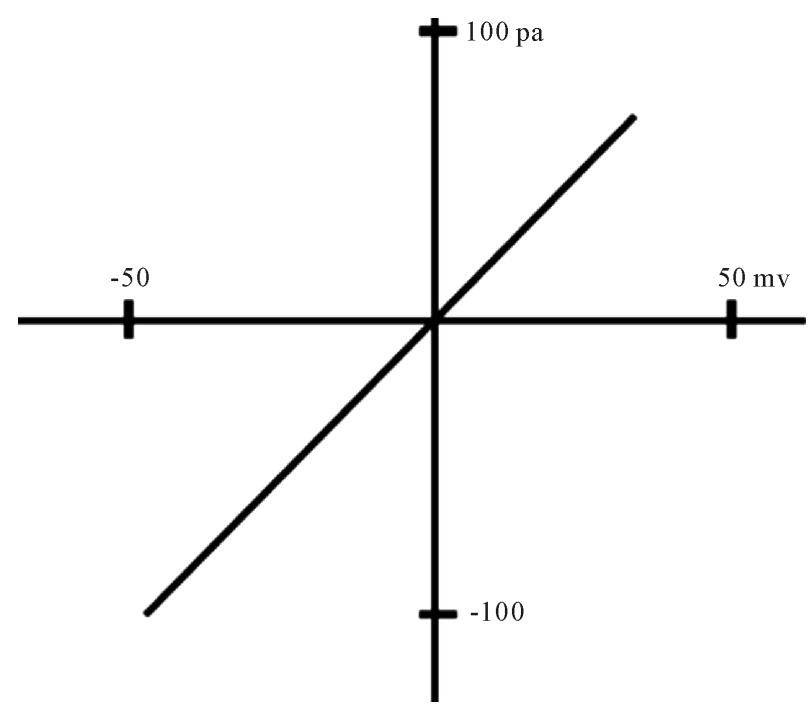

Figure 5. Shows the results of an experiment to determine reversal potential. It plots transduction current versus membrane voltage while a fixed mechanical input is applied to the hair cell It is abstracted from Figure 2 of [4] and it shows that the reversal potential of the hair cell is zero.

\section{Results}

The observations and interpretations made here are a serious attempt at reverse engineering.

The concepts reviewed here point out:

1) Mechanical motion of the hair cell cilia can change cilia spacing resulting in modulation of the membrane capacity and consequently the membrane voltage of the cell.

2) Transduction that is independent of ion type corroborates a capacitive transduction hypothesis.

3) A transduction reversal potential of zero corroborates a capacitive transduction hypothesis.

4) The positive endolymph voltage enhances capacitive transduction.

5) Capacitive transduction is fast and is therefore compatible with the speed requirements of a hair cell transducer which must function at rates of up to $2 \times 10^{5} \mathrm{~Hz}$.

6) Supporting cells can contribute to hair cell sensitivity with capacitive transduction by reducing the unmodulated component of membrane capacity.

\section{Conclusions}

The Nernst equation defines the behavior of an ion pore in a membrane separating solutions of differing ionic concentrations. Published measurements show that the Nernst criteria are not met, thus falsifying the popular theory that mechanical to electrical transduction is the result of a mechanically modulated ion channel at the tip of the cilia [2]. This reopens the question that how the hair cell performs this mechanical to electrical transduction.

The corroborative evidence, listed above, presents a strong case for a hypothesis, presented in this paper, in which hair cells use capacitive transduction in the performance of their crucial functions of transducing mechanical vibrations into electrical signals to be processed by an animal's nervous system. Yet a theory of capacitive transduction is not proven by these corroborative inferences and will not be strongly affirmed, or falsified, until someone measures the in-phase and quadrature components of hair cell membrane impedance during transduction.

In our effort to fathom the functionality of the hair cell, we cannot do better than to follow the admonition of Lao Tzu [6].

"As a thing the way is.

Shadowy, indistinct. 
Indistinct and shadowy,

Yet within it is an image;

Shadowy and indistinct,

Yet within it is substance.

Dim and dark,

Yet within it is an essence.

This essence is quite genuine

And within it is something that can be tested."

\section{References}

[1] Hudspeth, A.J., Choe, Y., Mehta, A.D. and Martin, P. (2000) Putting Ion Channels to Work: Mechanoelectrical Transduction, Adaptation and Amplification by Hair Cells. Proceedings of the National Academy Science of the United States of America, 97, 11765-11772. http://dx.doi.org/10.1073/pnas.97.22.11765

[2] Rossetto, M. (2013) Falsification of the Ionic Channel Theory of Hair Cell Transduction. Communicative Integrative Biology, 6, Article ID: e26763.

[3] Hille, B. (1992) Ionic Channels of Excitable Membranes. Sinaur Press, Sunderland Mass.

[4] Corey, D.P. and Hudspth, A.J. (1979) Ionic Basis of the Receptor Potential in a Vertebrate Hair Cell. Nature, 381, 675-677. http://dx.doi.org/10.1038/281675a0

[5] Holton, T. and Hudspth, A.J. (1986) The Transduction Channel of Hair Cells from the Bull Frog Characterized by Noise Analysis. Journal of Physics, 375, 195-227.

[6] Tzu, L. (1963) Tao Te Ching. Penguin Putnam, New York, Xvi. 